University of New Hampshire

University of New Hampshire Scholars' Repository

Physics Scholarship

Physics

2014

\title{
Excitation of EMIC waves detected by the Van Allen Probes on 28 April 2013
}

J. C. Zhang

University of New Hampshire

A A. Saiken

University of New Hampshire - Main Campus

Lynn M. Kistler

University of New Hampshire, lynn.kistler@unh.edu

Charles W. Smith

University of New Hampshire - Main Campus, charles.smith@unh.edu

Harlan E. Spence

University of New Hampshire, harlan.spence@unh.edu

See next page for additional authors

Follow this and additional works at: https://scholars.unh.edu/physics_facpub

Part of the Physics Commons

\section{Recommended Citation}

Zhang, J.-C., Saikin, A.A., Kistler, L.M., Smith, C.W., Spence, H.E., Mouikis, C.G., Torbert, R.B., Larsen, B.A., Reeves, G.D., Skoug, R.M., Funsten, H.O., Kurth, W.S., Kletzing, C.A., Allen, R.C., Jordanova, V.K. Excitation of EMIC waves detected by the Van Allen Probes on 28 April 2013. (2014) Geophysical Research Letters, 41 (12), pp. 4101-4108. doi:10.1002/2014GL060621

This Article is brought to you for free and open access by the Physics at University of New Hampshire Scholars' Repository. It has been accepted for inclusion in Physics Scholarship by an authorized administrator of University of New Hampshire Scholars' Repository. For more information, please contact Scholarly.Communication@unh.edu. 


\section{Authors}

J. C. Zhang, A A. Saiken, Lynn M. Kistler, Charles W. Smith, Harlan E. Spence, Christoforos Mouikis, Roy B. Torbert, Bradford Larsen, Geoffrey Reeves, R. M. Skoug, H. O. Funsten, W. S. Kurth, C A. Kletzing, R. C. Allen, and V K. Jordanova 


\section{Geophysical Research Letters}

\section{RESEARCH LETTER}

10.1002/2014GL060621

\section{Key Points:}

- Three EMIC wave events are observed only on Van Allen Probe-A

- Three overlapping $\mathrm{H}^{+}$populations are present when the waves are observed - Linear theory predicts the excitation of the waves with elevated thresholds

Correspondence to:

J.-C. Zhang,

jichun.zhang@unh.edu

\section{Citation:}

Zhang, J.-C., et al. (2014), Excitation of EMIC waves detected by the Van Allen Probes on 28 April 2013, Geophys. Res. Lett., 41, 4101-4108, doi:10.1002/ 2014 GL060621.

\section{Received 23 MAY 2014} Accepted 3 JUN 2014

Accepted article online 6 JUN 2014

Published online 27 JUN 2014

\section{Excitation of EMIC waves detected by the Van Allen Probes on 28 April 2013}

\author{
J.-C. Zhang ${ }^{1}$, A. A. Saikin ${ }^{1}$, L. M. Kistler ${ }^{1}$, C. W. Smith ${ }^{1}$, H. E. Spence ${ }^{1}$, C. G. Mouikis ${ }^{1}$, R. B. Torbert ${ }^{1}$, \\ B. A. Larsen ${ }^{2}$, G. D. Reeves ${ }^{2}$, R. M. Skoug ${ }^{2}$, H. O. Funsten ${ }^{2}$, W. S. Kurth ${ }^{3}$, C. A. Kletzing ${ }^{3}$, R. C. Allen ${ }^{4,5}$, \\ and V. K. Jordanova ${ }^{2}$ \\ ${ }^{1}$ Space Science Center, University of New Hampshire, Durham, New Hampshire, USA, ${ }^{2}$ Los Alamos National Laboratory, Los Alamos, \\ New Mexico, USA, ${ }^{3}$ Department of Physics and Astronomy, University of lowa, lowa City, lowa, USA, ${ }^{4}$ Physics and Astronomy \\ Department, University of Texas at San Antonio, San Antonio, Texas, USA, ${ }^{5}$ Space Science and Engineering Division, Southwest \\ Research Institute, San Antonio, Texas, USA
}

\begin{abstract}
We report the wave observations, associated plasma measurements, and linear theory testing of electromagnetic ion cyclotron (EMIC) wave events observed by the Van Allen Probes on 28 April 2013. The wave events are detected in their generation regions as three individual events in two consecutive orbits of Van Allen Probe-A, while the other spacecraft, $B$, does not detect any significant EMIC wave activity during this period. Three overlapping $\mathrm{H}^{+}$populations are observed around the plasmapause when the waves are excited. The difference between the observational EMIC wave growth parameter $\left(\Sigma_{h}\right)$ and the theoretical EMIC instability parameter $\left(S_{h}\right)$ is significantly raised, on average, to $0.10 \pm 0.01,0.15 \pm 0.02$, and $0.07 \pm 0.02$ during the three wave events, respectively. On Van Allen Probe-B, this difference never exceeds 0 . Compared to linear theory $\left(\Sigma_{h}>S_{h}\right)$, the waves are only excited for elevated thresholds.
\end{abstract}

\section{Introduction}

Electromagnetic ion cyclotron (EMIC) waves play an important role in the overall dynamics of the Earth's magnetosphere, contributing to the energization and loss of magnetospheric particles. EMIC waves are typically excited by an anisotropic $\left(T_{\perp}>T_{/ /}\right)$distribution of energetic ( 10-100 keV) $\mathrm{H}^{+}$[e.g, Kennel and Petschek, 1966; Anderson et al., 1996]. The hot $\mathrm{H}^{+}$distribution, with a temperature anisotropy sufficiently high to satisfy the EMIC instability, is called the wave free-energy provider [e.g., Cornwall, 1965; Rauch and Roux, 1982]. Besides contributing to the increase of the EMIC wave growth rate, energetic $\mathrm{H}^{+}$are able to greatly modify the real part of the wave dispersion relation, dramatically changing the wave generation [e.g., Arnoldy et al., 2005; Engebretson et al., 2007; Gamayunov and Khazanov, 2008].

Under a dipole-like magnetic field configuration in the inner magnetosphere, the excitation region of EMIC waves is usually near the geomagnetic equator [e.g., Fraser and Nguyen, 2001], where the larger total plasma density and lower magnetic field strength $(|B|)$ increase the wave growth rate [Kennel and Petschek, 1966]. EMIC waves are preferentially generated in regions where hot anisotropic $\mathrm{H}^{+}$and cold dense ion populations spatially overlap [e.g., Jordanova et al., 2001; Pickett et al., 2010; Zhang et al., 2011]. In the terrestrial magnetosphere, these ion populations can commonly be found where the ring current overlaps the plasmasphere or plasmaspheric plumes [Gurgiolo et al., 2005; Thorne, 2010].

In this study, we report EMIC wave events and associated local plasma conditions observed by the Van Allen Probes on 28 April 2013. The two-point and frequent in situ wave and plasma measurements in almost the same region provide unprecedented insight into the excitation of EMIC waves. After instrumentation in the next section, we present wave and plasma observations for the events and comparison with linear theory.

\section{Instrumentation}

The Van Allen Probes mission, formerly known as the Radiation Belt Storm Probes (RBSP) mission [Kessel et al., 2013; Mauk et al., 2013], consists of two spacecraft in nearly identical highly elliptical, low-inclination $\left(\sim 10^{\circ}\right)$ orbits with a perigee of 1.1 Earth radii $\left(R_{E}\right)$, an apogee of $5.8 R_{E}$, and a period of $\sim 9 \mathrm{~h}$. The perigee-apogee line, i.e., the line of apsides, precesses in local time at a rate of $\sim 210 \% \mathrm{yr}$. The precise orbits of the two probes are slightly different, and one probe laps the other every $\sim 2.5$ months. As a result, the interspacecraft distance 

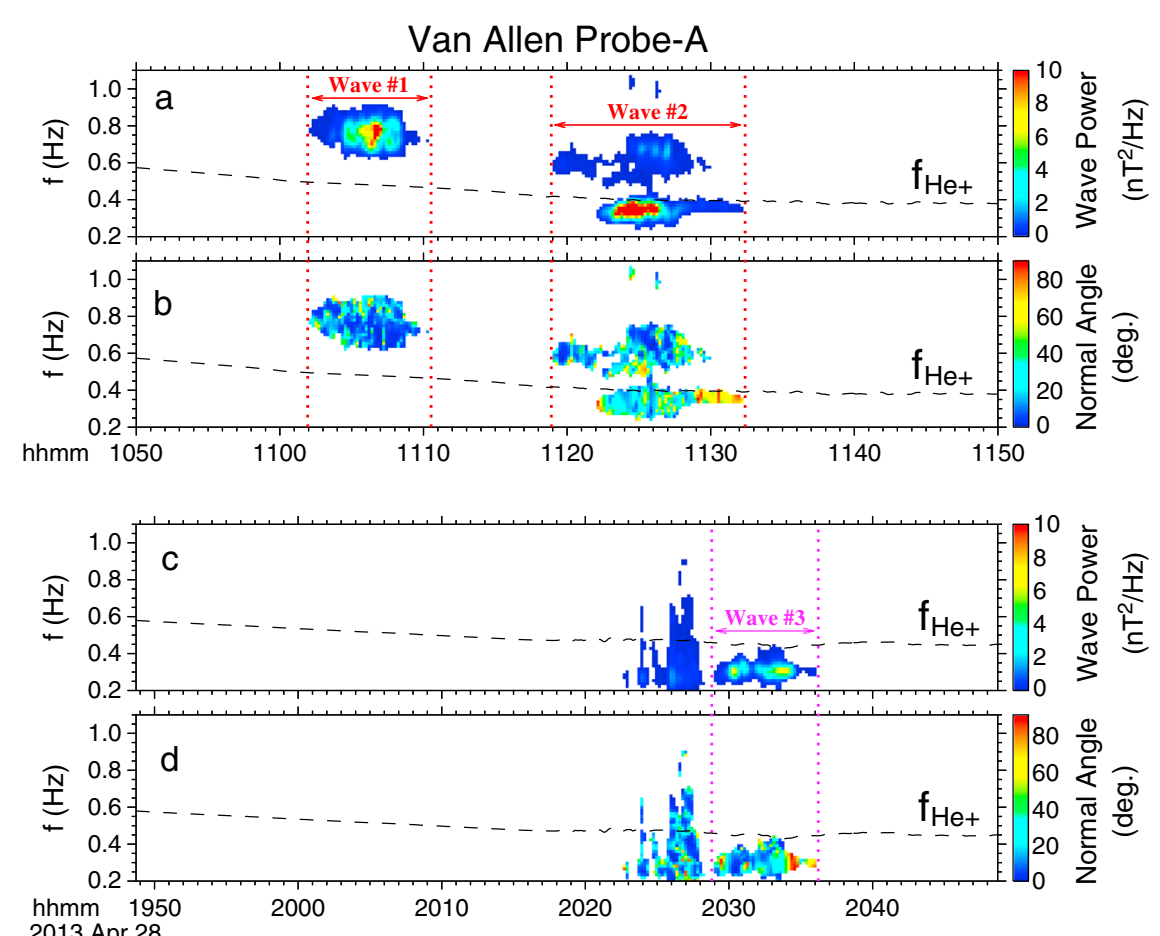

Figure 1. EMIC wave activity detected by EMFISIS on Van Allen Probe-A during (a and b) 1050-1150 UT on 28 April 2013 and during the corresponding time period on the next orbit, i.e., (c and d) 1948:42-2048:42 UT on 28 April 2013.

Figures $1 \mathrm{a}$ and $1 \mathrm{c}$ (Figures $1 \mathrm{~b}$ and $1 \mathrm{~d}$ ) show frequency-UT spectrograms of wave power (wave normal angle). Three pairs of the vertical dotted lines indicate the start and end times of the three wave events. The dashed black trace in each panel represents local $\mathrm{He}^{+}$gyrofrequencies $\left(f_{\mathrm{He}+}\right)$.

periodically varies between $\sim 0.1$ and $\sim 5 R_{E}$, and resampling times for a specific region of the magnetosphere vary anywhere from minutes to $4.5 \mathrm{~h}$. A comprehensive suite of instruments, identical on the two Van Allen Probes (designated as A and B), measures a variety of parameters in particles (electrons, ions, and ion composition), magnetic field (B), electric field ( $\mathbf{E})$, and waves ( $\mathbf{\delta} \mathbf{E}$ and $\boldsymbol{\delta} \mathbf{B})$.

High temporal resolution (64 vectors/s) magnetic field measurements are obtained from the magnetometer instrument in the Electric and Magnetic Field Instrument Suite and Integrated Science (EMFISIS) [Kletzing et al., 2013] on board the Van Allen Probes, and the wave frequency spectra of the measurements are derived using the fast Fourier transform (FFT) technique (see section 3.1). The EMFISIS magnetometer measures DC magnetic fields, and the Waves instrument collects an extensive set of wave electric and magnetic field measurements with frequencies from $10 \mathrm{~Hz}$ to $12 \mathrm{kHz}$, and up to $400 \mathrm{kHz}$ for single-axis electric field. Each EMFISIS instrument consists of a triaxial fluxgate magnetometer (MAG) and a triaxial magnetic search coil magnetometer. Wave electric fields are obtained using the triaxial electric field booms from the Electric Fields and Waves instrument.

Plasma data during the EMIC wave events are obtained from the Helium, Oxygen, Proton, and Electron (HOPE) mass spectrometer [Funsten et al., 2013], part of the Radiation Belt Storm Probes-Energetic Particle Composition and Thermal Plasma (RBSP-ECT) instrument suite [Spence et al., 2013]. Using an electrostatic top-hat analyzer and time-offlight technology, the HOPE mass spectrometer measures electron and ion flux distributions over $4 \pi \mathrm{sr}$ every spacecraft spin in the energy range of $\sim 1 \mathrm{eV}-52 \mathrm{keV}$ and distinguishes the three major ion species: $\mathrm{H}^{+}, \mathrm{He}^{+}$, and $\mathrm{O}^{+}$.

\section{EMIC Waves on 28 April 2013}

\subsection{Wave Observations}

Right after the recovery phase of a weak geomagnetic storm (minimum Dst $=-49 \mathrm{nT}$ at $2100 \mathrm{UT}, 24$ April 2013), three distinct EMIC wave events were detected by the EMFISIS magnetometer instrument on Van Allen Probe-A on 28 April 2013. Figure 1 shows the wave power and wave normal angle spectra from the FFT analysis of the EMFISIS magnetic field measurements, using 1024 time points and a step length of 128 points. 


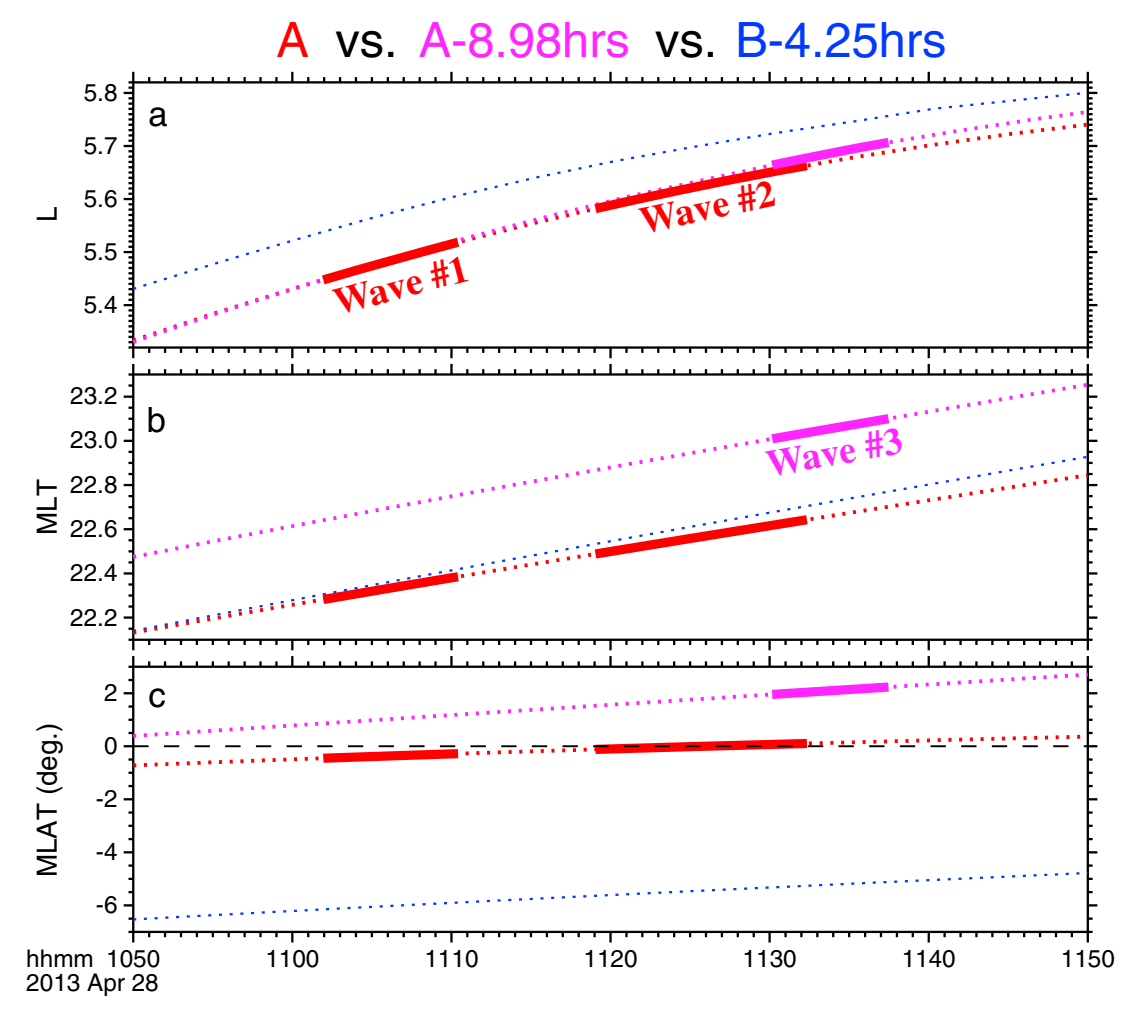

Figure 2. Position comparison of the Van Allen Probes $A$ and B in L, MLT, and MLAT. The red lines are for those of $A$ between 1050 and 1150 UT on 28 April 2013, magenta for $A$ on the next orbit but shifted backward in UT by $8.98 \mathrm{~h}$, and blue for B but shifted backward in UT by $4.25 \mathrm{~h}$. The periods corresponding to the observation of the three wave events shown in Figure 1 are marked with the bold solid lines. The horizontal dashed black line in the last panel represents $0^{\circ}$ magnetic latitude.

Data for wave powers less than $0.05 \mathrm{nT}^{2} / \mathrm{Hz}$ are removed from the plot. The observations from the same arc segments of two consecutive orbits are shown, with the first orbit in Figures $1 \mathrm{a}$ and $1 \mathrm{~b}$ and the second orbit in Figures $1 \mathrm{c}$ and $1 \mathrm{~d}$. The difference of their plotted start time is determined by the time difference in the two consecutive perigees, i.e., $8 \mathrm{~h} 58 \mathrm{~m} 42 \mathrm{~s}$. As indicated with the vertical dotted lines in Figure 1, the intervals of substantial wave activity are labeled Wave \#1, \#2, and \#3, which start at 1101:56, 1118:54, and 2028:48 UT and last for $8 \mathrm{~m} 36 \mathrm{~s}, 13 \mathrm{~m} 30 \mathrm{~s}$, and $7 \mathrm{~m} 25 \mathrm{~s}$, respectively. The local He${ }^{+}$gyrofrequencies $\left(f_{\mathrm{He}+}=q B / m_{\mathrm{He}+}\right)$ are represented by the dashed black trace in each panel. The gyrofrequencies $f_{\mathrm{H}+}$ and $f_{\mathrm{O}+}$, which are four times higher and lower than $f_{\mathrm{He}+}$, respectively, lie outside of the plotted frequency range, i.e., $0.2-1.1 \mathrm{~Hz}$, and are thus not shown.

In addition to differences in wave amplitude, the three wave events demonstrate differences in wave frequencies. Wave \#1 is always above $f_{\mathrm{He}+}$. Thus, it is in the $\mathrm{H}^{+}$band. Wave \#2 includes both the $\mathrm{H}^{+}$and $\mathrm{He}^{+}$bands, but Wave \#3 is only in the $\mathrm{He}^{+}$band. Note that the broadband wave activity before Wave \#3 is due to non-EMIC geomagnetic pulsations [Anderson, 1993]. The wave normal angle of all the three wave events is predominantly small. Their ellipticity (not shown) is normally negative, indicating a left-handed wave polarization.

While Van Allen Probe-A clearly observed the three wave events, B, trailing A by $4.25 \mathrm{~h}$, did not detect any significant EMIC wave activity $\left(\geq 0.05 \mathrm{nT}^{2} / \mathrm{Hz}\right)$ in almost the same region. Figure 2 shows the relative positions of the spacecraft in $L$, magnetic local time (MLT), and magnetic latitude (MLAT) for $1 \mathrm{~h}$. The $L$ value (= L_TS04) is computed with the Tsyganenko and Sitnov empirical magnetic field model (TS04) [Tsyganenko and Sitnov, 2005]. The three intervals of significant EMIC wave activity are indicated with the bold, solid lines. For the purpose of comparison, the universal time (UT) of A during the orbit of the observation of Wave \#3 and the immediately preceding orbit of B are shifted backward in UT by the orbital period $(8.98 \mathrm{~h}$ for $\mathrm{A})$ and the difference in perigee time between $A$ and $B(4.25 \mathrm{~h}$ for $B)$, respectively. Thus, Figure 2 shows three overlapping orbital tracks: two for the sequential orbits of $A$ and one for $B$.

The red and magenta lines in Figure 2, comparing the two consecutive orbits of A, show that their $L$ values are almost identical, MLT has a difference of $\sim 0.4$ (due to the MLT precession of the spacecraft), and MLAT differs 

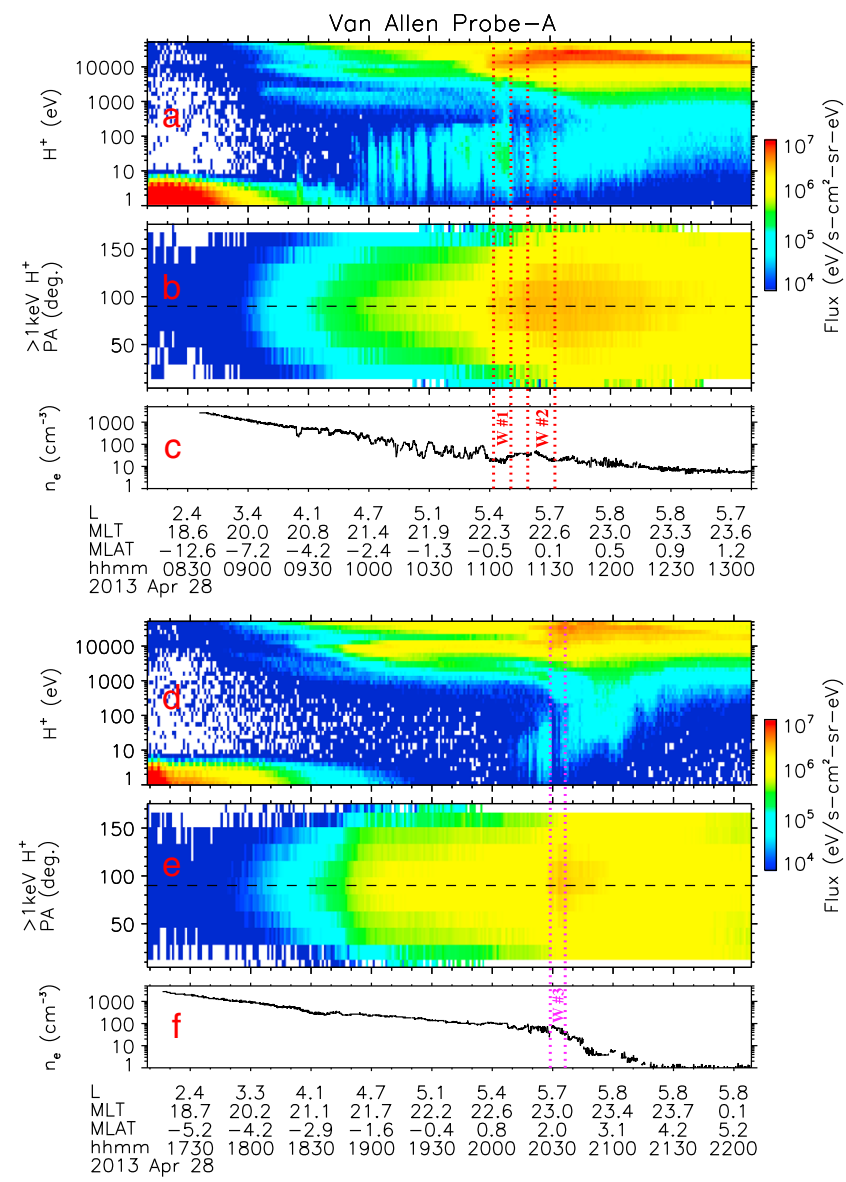

Figure 3. HOPE $\mathrm{H}^{+}$flux spectrograms and EMFISIS electron density $\left(n_{e}\right)$ on the Van Allen Probe-A (a-c) during 0810-1310 UT on 28 April 2013 and during the corresponding time period on the next orbit, i.e., (d-f) 1708:42-2208:42 UT on 28 April 2013. Figures $3 a$ and $3 d$ (Figures $3 b$ and 3e) show energy-UT ( $>1 \mathrm{keV} \mathrm{H}^{+}$pitch angle- or PA-UT) spectrograms. Three pairs of the vertical dotted lines indicate the start and end time of the three wave events, respectively. In Figures $3 \mathrm{~b}$ and $3 \mathrm{e}$, the horizontal dashed lines mark $90^{\circ}$ pitch angle. Listed below Figures $3 \mathrm{c}$ and $3 \mathrm{f}$ are $L$ (= L_TS04), magnetic local time (MLT, hours), magnetic latitude (MLAT, degrees), and universal time (UT, hours). from each other in the range of $1.3^{\circ}-2.0^{\circ}$ during the wave events. Regarding the spatial difference between $A$ and $B$, the $L$ value of the blue line $(B-4.25 \mathrm{~h})$ is greater than that of the red line (A) by $<0.1$ and their MLT are nearly the same. However, B's MLAT are different from A's by at least $4.5^{\circ}$. $B$ is always in the Southern Hemisphere (MLAT $<0)$, but A crossed the magnetic equatorial plane $(M L A T=0)$ while observing Wave \#2.

\subsection{Local Plasma Conditions}

Figure 3 shows $\mathrm{H}^{+}$energy-time and pitch angle (PA)-time flux spectrograms from HOPE and the total electron density, $n_{e}$, from EMFISIS on $A$ in the orbits of Wave \#1 and Wave \#2 (Figures 3a-3c) and Wave \#3 (Figures 3d-3f). Derived from the frequency of the upper hybrid waves (UHWs) observed by the EMFISIS Waves instrument is $n_{e}$. Data gaps in $n_{e}$ result from no clearly identifiable UHW activity. The plot time intervals are similar to Figure 1 but for a longer period of $5 \mathrm{~h}$. The ephemeris parameters of the spacecraft shown in Figure 2, i.e., L, MLT, MLAT, and UT, are included at the bottom of the figure. Measurements at $B$ (not shown) are qualitatively similar to $A$, but a quantitative analysis (see section 3.3) reveals that local plasma conditions at $B$ are not sufficiently EMIC wave favorable to drive wave activity.

Enhanced $\mathrm{H}^{+}$populations are present in three distinct energy ranges around the EMIC wave events:

1. $>1 \mathrm{keVH}^{+}$are freshly injected from the plasma sheet. Their spectra demonstrate "nose-like" structures [e.g., Smith and Hoffman, 1974; Vallat et al., 2007], characteristic of deeper access to the inner magnetosphere at a specific energy. This unique spectral feature often results from comparable westward and eastward components of the $\mathbf{E} \times \mathbf{B}$ and gradient-curvature drifts, resulting in more inward injections of those particular particles.

2. $<10 \mathrm{eV} \mathrm{H}^{+}$are an intense population in the HOPE energy range only in the first $\sim 2 \mathrm{~h}$ of the plotted period, soon after perigee. This is the high-energy tail of the plasmaspheric population.

3. $\sim 2-300 \mathrm{eV} \mathrm{H}^{+}$exist from $\sim 0955$ UT in Figure 3a and from $\sim 2015$ UT in Figure $3 \mathrm{~d}$. The population lies in the transition region from the cold plasmasphere to the hot ring current, and its association with the observed EMIC waves is not clear. Nevertheless, their flux variation over UT anticorrelates with $n_{e}$. That is, higher $\mathrm{H}^{+}$ fluxes are observed at times of lesser $n_{e}$ or lower frequencies $(20-500 \mathrm{kHz})$ of UHWs (not shown). As a result, this $\mathrm{H}^{+}$population is likely related to the UHW activity during the periods.

Although the $\mathrm{H}^{+}$spectral features changed noticeably over the two orbits (Figures 3a versus 3d), the fluxes of $>1 \mathrm{keV} \mathrm{H}^{+}$are always enhanced around $\mathrm{PA}=90^{\circ}$ during the wave events (Figures $3 \mathrm{~b}$ and $3 \mathrm{e}$ ). Note that the dominance of the perpendicular fluxes over parallel even continues after each wave event. 

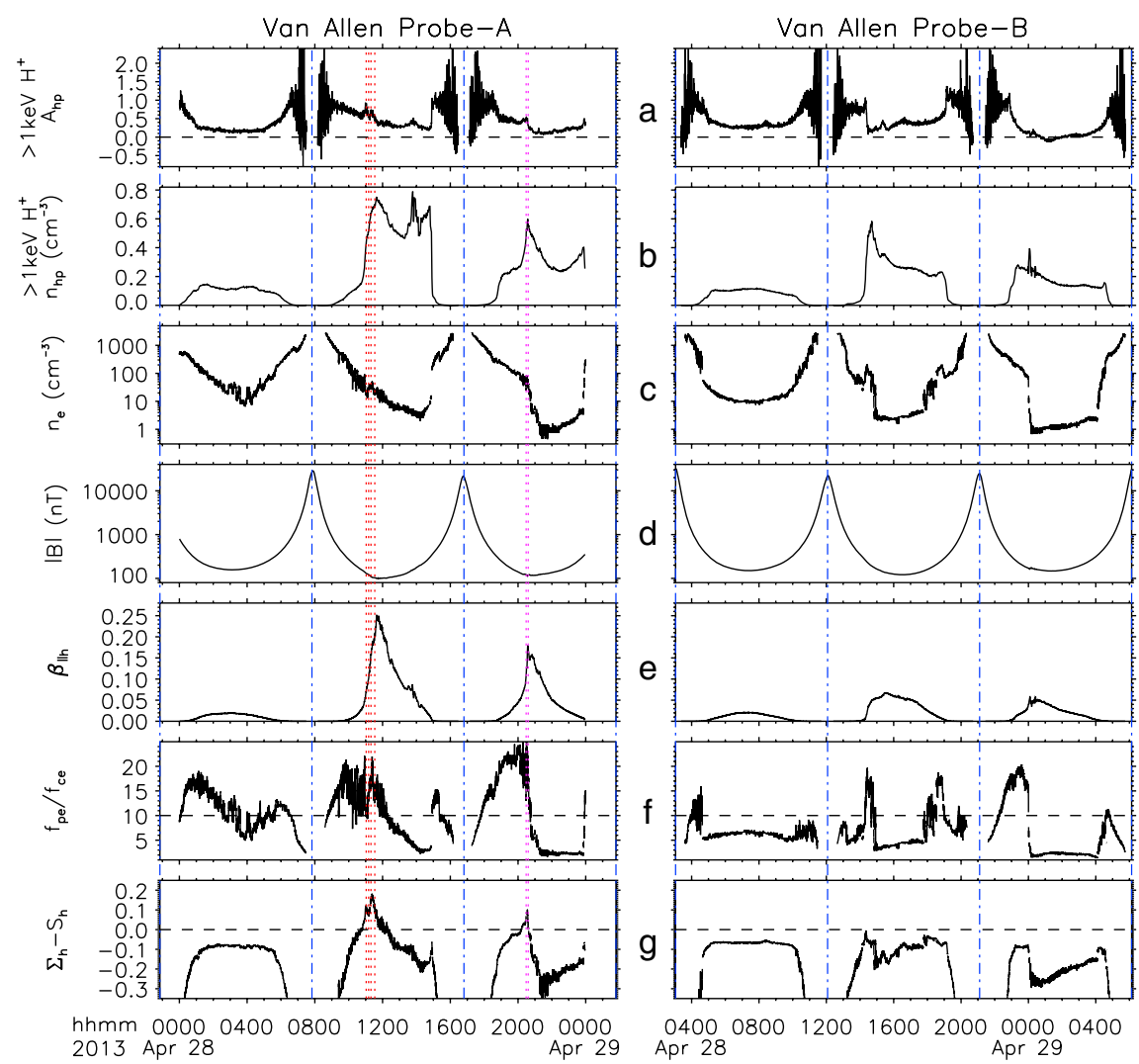

Figure 4. EMIC instability related parameters on (left) Van Allen Probes-A and (right) $B$ in three consecutive orbits on 27-29 April 2013. From top to bottom, the panels show hot-proton $(>1 \mathrm{keV})$ temperature anisotropy $\left(A_{\mathrm{hp}}=T_{\perp} / T_{\|}-1\right)$, hot-proton density $\left(n_{\mathrm{hp}}\right)$, electron density $\left(n_{e}\right)$, magnetic field magnitude $(|B|)$, hot-proton parallel plasma beta $\left(\beta_{\| h}\right)$, electron plasma/gyrofrequency ratio $\left(f_{\mathrm{pe}} / f_{\mathrm{ce}}\right)$, and the difference between the observational EMIC wave growth parameter $\left(\Sigma_{h}\right)$ and the theoretical EMIC instability parameter $\left(S_{h}\right)$. Three pairs of the vertical dotted lines indicate the start and end times of the three wave events, respectively. In Figures $4 \mathrm{a}, 4 \mathrm{f}$, and $4 \mathrm{~g}$, the horizontal dashed line marks the values of 0,10 , and 0 , respectively. The vertical dash-dotted blue lines denote the time of the spacecraft perigee passes. The plotted period starts and ends at two perigees.

Consistent with the spectrograms in Figures $3 a$ and $3 d$, the magnitude and variability of $n_{e}$ indicate that the waves occurred near the plasmapause. While Wave \#3 is at a distinct boundary with a sudden, large drop in $n_{e}$ the $n_{e}$ gradient when Waves \#1 and \#2 are observed is more gradual. However, all the three wave events are associated with decreases in $n_{e}$ or with negative $n_{e}$ gradients (less clear in Wave \#1 than the other two wave events).

\subsection{Linear Theory Testing}

To quantitatively evaluate conditions for the excitation of the EMIC waves, we test the linear theory of the EMIC instability with local magnetic field and plasma parameters, which are derived from the $\mathrm{H}^{+}$energy and PA fluxes. Based on linear theory, EMIC wave activity can occur only if $\Sigma_{h}>S_{h}$ [Gary et al., 1994; MacDonald et al., 2010], where the theoretical EMIC instability parameter

$$
S_{h}=\sigma_{0}+\sigma_{1} \ln \left(\frac{n_{\mathrm{hp}}}{n_{e}}\right)+\sigma_{2}\left[\ln \left(\frac{n_{\mathrm{hp}}}{n_{e}}\right)\right]^{2} .
$$

The number density of energetic $\mathrm{H}^{+}$(denoted by "hp," i.e., hot protons) is $n_{\mathrm{hp}}$. The electron density is $n_{e}$. The constants $\sigma_{0}=0.429, \sigma_{1}=0.124$, and $\sigma_{2}=0.0118$ were derived by Blum et al. [2009] by assuming that the local wave growth rate $\left(\gamma / \Omega_{p}\right)$ is 0.001 and fitting linear theory results to Los Alamos National Laboratory (LANL) Magnetospheric Plasma Analyzer observations at geosynchronous orbit (GEO). Those constants are still valid at places other than GEO. The observational EMIC growth parameter

$$
\Sigma_{h}=\left(\frac{T_{\perp}}{T_{\|}}-1\right) \beta_{\| h}^{\alpha_{h}},
$$


Table 1. Average Parameter Values With Standard Deviations During Wave Events

\begin{tabular}{lccccccc} 
Wave \# & $A_{\mathrm{hp}}$ & $n_{\mathrm{hp}}\left(\mathrm{cm}^{-3}\right)$ & $n_{e}\left(\mathrm{~cm}^{-3}\right)$ & $B(\mathrm{nT})$ & $\beta_{\| h}$ & $f_{\mathrm{pe}} / f_{\mathrm{ce}}$ & $\Sigma_{h}-S_{h}$ \\
\hline 1 & $0.70 \pm 0.10$ & $0.48 \pm 0.02$ & $21.17 \pm 4.72$ & $125.89 \pm 2.48$ & $0.09 \pm 0.01$ & $11.66 \pm 1.43$ & $0.10 \pm 0.01$ \\
2 & $0.61 \pm 0.06$ & $0.67 \pm 0.02$ & $29.92 \pm 8.73$ & $105.39 \pm 1.82$ & $0.18 \pm 0.01$ & $16.48 \pm 2.27$ & $0.15 \pm 0.02$ \\
3 & $0.46 \pm 0.07$ & $0.55 \pm 0.03$ & $54.79 \pm 14.90$ & $116.82 \pm 2.27$ & $0.15 \pm 0.02$ & $20.14 \pm 2.72$ & $0.07 \pm 0.02$ \\
\hline
\end{tabular}

where

$$
\alpha_{h}=a_{0}-a_{1} \ln \left(\frac{n_{\mathrm{hp}}}{n_{e}}\right)-a_{2}\left[\ln \left(\frac{n_{\mathrm{hp}}}{n_{e}}\right)\right]^{2}
$$

and $a_{0}=0.409, a_{1}=0.0145$, and $a_{2}=0.00028$ [Blum et al., 2009]. The parallel plasma beta of energetic $\mathrm{H}^{+} \beta_{\| h}=2 \mu_{0} n_{\mathrm{hp}} k T_{\|} / B^{2}$.

Figure 4 shows several associated parameters from both of the Van Allen Probes in three sequential orbits on 27-29 April 2013: temperature anisotropy for $>1 \mathrm{keV} \mathrm{H}^{+}, A_{\mathrm{hp}}=T_{\perp} / T_{\|}-1$ (Figure 4a), $n_{\mathrm{hp}}$ for $>1 \mathrm{keV} \mathrm{H}^{+}$ (Figure $4 b), n_{e}$ (Figure 4c), the local magnetic field magnitude $|B|$ (Figure $4 \mathrm{~d}$ ), $\beta_{\| h}$ for $>1 \mathrm{keV} \mathrm{H}^{+}$(Figure $4 \mathrm{e}$ ), the electron plasma/gyrofrequency ratio, $f_{\text {pe }} / f_{\text {ce }}$ (Figure $4 f$ ), and the difference between $\Sigma_{h}$ and $S_{h}$ (Figure $4 \mathrm{~g}$ ). $f_{\mathrm{pe}} / f_{\mathrm{ce}}=321 n_{e}{ }^{1 / 2}|B|^{-1}$, where $n_{e}$ is in $\mathrm{cm}^{-3}$ and $|B|$ in $\mathrm{nT}$. The $\mathrm{H}^{+}$moments $\left(T_{\perp}, T_{\|}\right.$, and $\left.n_{\mathrm{hp}}\right)$ are calculated with $\mathrm{H}^{+} 3-\mathrm{D}$ distributions measured by HOPE in energy channels over $1 \mathrm{keV}$ ( $970 \mathrm{eV}$, to be precise). For the purpose of comparison, the $y$ axis range of each panel is kept the same for the two spacecraft. Again, the start and end time of the wave events are denoted by the three pairs of the vertical dotted red lines. Table 1 lists the averages and standard deviations of the parameters during the wave events.

The mean value of $A_{\mathrm{hp}}$ is moderately high during the wave events, with the maximum average value at $0.70 \pm 0.10$ in Wave \#1. Note that large $A_{\text {hp }}$ spikes around perigee (denoted by the vertical dash-dotted blue lines) are a computational artifact resulting from the low abundance (and thus low count rates) of $\mathrm{H}^{+}$at energies $>1 \mathrm{keV}$ (see Figures $3 \mathrm{a}$ and $3 \mathrm{~d}$ ). During the periods of the waves $n_{\mathrm{hp}}$ is apparently enhanced, although it is not at its maximum. During the wave events, $n_{e}$ is greater than the density of $\mathrm{H}^{+}$in the full energy range of HOPE by a factor of 6-110 (not shown), indicating a dense plasma population below the lower energy limit of HOPE. The three wave events occurred at low MLATs, close to local minimum $|B|$ regions (i.e., on the magnetic equatorial plane). The averages of $|B|$ are $125.89 \pm 2.48,105.39 \pm 1.82$, and $116.82 \pm 2.27 \mathrm{nT}$ in the three events, respectively. During the first two wave events, $\beta_{\| h}$ is not at a maximum value but is clearly enhanced. Notably, the third wave event does occur at a local maximum of $\beta_{\| h}$. Associated with the characteristic energy for cyclotron interactions $\left(=|B|^{2} /\left(2 \mu_{0} k n_{e}\right)\right)\left[\right.$ Kennel and Petschek, 1966], $f_{\mathrm{pe}} / f_{\mathrm{ce}}$ is expected to exceed 10 for the excitation of EMIC waves [Chen et al., 2009; Zhang et al., 2011]. As listed in Table 1, the wave event averages of $f_{\mathrm{pe}} / f_{\mathrm{ce}}$ are all above 10 . However, they are also greater than 10 in several time intervals when no EMIC wave activity is present (Figure 4f). We use $\Sigma_{h}-S_{h}$ as a more comprehensive proxy for EMIC wave generation. While a positive value of $\Sigma_{h}-S_{h}$ is not always accompanied by EMIC wave activity, the average of $\Sigma_{h}-S_{h}$ is clearly elevated during each wave event, with positive values of $0.10 \pm 0.01,0.15 \pm 0.02$, and $0.07 \pm 0.02$, respectively. On Van Allen Probe-B, $\Sigma_{h}-S_{h}$ never exceeds 0 .

\section{Discussion and Conclusions}

We report the wave observations, associated plasma measurements, and linear theory testing of EMIC waves observed by the Van Allen Probes on 28 April 2013. Although EMIC waves have been intensively investigated [e.g, Kennel and Petschek, 1966; Young et al., 1981; Rauch and Roux, 1982; Roux et al., 1982; Anderson et al., 1996; Fraser and Nguyen, 2001; Halford et al., 2010; Pickett et al., 2010; Zhang et al., 2010, 2011; Min et al., 2012; Allen et al., 2013; Lin et al., 2014], plasma properties relevant to EMIC wave excitation have not been fully understood in the magnetosphere due to the wide spatial extent of EMIC wave generation and propagation. For example, Lin et al. [2014] recently found that the correlation of positive $A_{\text {hp }}$ events, EMIC instability threshold $\left(\Sigma_{h}>S_{h}\right)$, and EMIC waves observed by the polar-orbiting Cluster spacecraft is low. The two-point, frequent measurements of the Van Allen Probes allow us to pinpoint local field and plasma conditions for EMIC waves in the inner magnetosphere. New insight into the excitation of EMIC waves from this report is as follows: 
1. EMIC waves are detected during three individual events in two consecutive orbits of Van Allen Probe-A, while B detects no significant EMIC wave activity during the period. The wave events are apparently localized in or near their generation regions when observed, because they still have low wave normal angles and left-hand polarizations [Kennel and Petschek, 1966; Roux et al., 1982]. The local wave occurrences are reconfirmed with the testing of linear theory, even though it has intrinsic limitations (see below). No EMIC wave activity is observed at B because local plasma conditions are not favorable for EMIC wave excitation. For example, $\beta_{\| h}$ is low throughout the time interval and $\Sigma_{h}-S_{h}$ never reaches a large, positive value that is close to those at $A$ during the wave periods. This could be due to the more offequatorial position of $B$ during the wave observations at $A$. In other words, $B$ is at least $4.5^{\circ}$ from $A$ in MLAT.

2. Three overlapping $\mathrm{H}^{+}$populations around the plasmapause are present during the EMIC wave events: freshly injected hot $\mathrm{H}^{+}$from the plasma sheet $(>1 \mathrm{keV})$, cold $\mathrm{H}^{+}$originated from the plasmasphere $(<10 \mathrm{eV}$; most not observed by $\mathrm{HOPE})$, and $\mathrm{H}^{+}$in the transition region from the plasmasphere to the ring current ( 2-300 eV; likely related to UHWs). The waves occurred in the preferred EMIC wave excitation region, i.e., near the plasmapause [e.g., Fraser and Nguyen, 2001], and the waves are driven by energetic $\mathrm{H}^{+}$ in so-called nose-like spectral structures. This $\mathrm{H}^{+}$population resides near the ion injection boundary on the nightside, specifically, 1-2 h before the local midnight. Note that there also exist "nose" structures at Van Allen Probe-B, but no EMIC waves are ever observed on that spacecraft during the period.

3. Linear theory indicates that $\Sigma_{h}-S_{h}$ is significantly raised, on average, to $0.10 \pm 0.01,0.15 \pm 0.02$, and $0.07 \pm 0.02$ during the three wave events, respectively. These are elevated thresholds for EMIC wave excitation with $\gamma / \Omega_{p}=0.001$. A positive value of $\Sigma_{h}-S_{h}$ is not always accompanied by EMIC wave activity. Even a large positive value of $\Sigma_{h}-S_{h}$ is not a sufficient condition for the excitation of an EMIC wave (e.g., after Wave \#2 in Figure 4g): Limitations in linear theory, such as simplifying the ion distribution as Maxwellian [Khazanov et al., 2007] and not considering heavy ions $\left(\mathrm{He}^{+}\right.$and $\left.\mathrm{O}^{+}\right)$and nonlinear effects, may be the reason for this. The wave-period enhancements of $\Sigma_{h}-S_{h}$ result from combined variations in $A_{\mathrm{hp}}, n_{\mathrm{hp}}, n_{e},|B|$, and $\beta_{\| h}$. Negative $n_{e}$ gradients might also play an important role in the generation of the waves. They could guide waves, maintain a small wave normal angle, and thus greatly increase the raypath-integrated wave gain [Chen et al., 2009].

Changes in the abundance ratios of $\mathrm{H}^{+}, \mathrm{He}^{+}$, and $\mathrm{O}^{+}$ions, dominated by those from the plasmasphere, probably cause the distinct band differences among the three EMIC wave events [Rauch and Roux, 1982; Chen et al., 2009]. A reproduction of the band features with a wave model is beyond the scope of the current investigation and will be a topic for future work. The present study serves as a foundation for a statistical examination of all EMIC wave events observed by the Van Allen Probes, providing a clearer understanding of plasma conditions for the excitation of them. Moreover, to achieve a better understanding of the generation and propagation of EMIC waves and their interactions with particles in the magnetosphere, it is necessary to conduct conjunction studies by adding balloon- or ground-based observations as well as in situ

Acknowledgments

This work was supported by RBSP-ECT funding provided by JHU/APL contract 967399 under NASA's Prime contract NAS5-01072 and by lowa subcontract 1000556126 to UNH in support of the Van Allen Probes and EMFISIS/MAG instruments. Work at UNH was also supported by NASA under grant numbers NNX11AO82G and NNX13AE23G. Work at LANL was performed under the auspices of the United States Department of Energy. Work at the University of lowa was performed under support on JHU/APL contract 921647 under NASA's Prime contract NAS501072. The authors thank RBSP team members for RBSP data preparation and software development. J.-C. Zhang thanks Brian Fraser and S. Peter Gary for helpful discussions.

The Editor thanks two anonymous reviewers for their assistance in evaluating this paper. measurements from other missions.

\section{References}

Allen, R. C., J.-C. Zhang, L. M. Kistler, H. E. Spence, R.-L. Lin, M. W. Dunlop, and M. Andre (2013), Multiple bidirectional EMIC waves observed by Cluster at middle magnetic latitudes in the dayside magnetosphere, J. Geophys. Res. Space Physics, 118, 6266-6278, doi:10.1002/jgra.50600.

Anderson, B. J. (1993), Statistical studies of Pc 3-5 pulsations and their relevance for possible source mechanisms of ULF waves, Ann. Geophys., 11, 2-3, doi:10.5194/angeo-24-3027-2006.

Anderson, B. J., R. E. Denton, G. Ho, D. C. Hamilton, S. A. Fuselier, and R. J. Strangeway (1996), Observational test of local proton cyclotron instability in the Earth's magnetosphere, J. Geophys. Res., 101(A10), 21,527-21,543, doi:10.1029/96JA01251.

Arnoldy, R. L., et al. (2005), Pc 1 waves and associated unstable distributions of magnetospheric protons observed during a solar wind pressure pulse, J. Geophys. Res., 110, A07229, doi:10.1029/2005JA011041.

Blum, L. W., E. A. MacDonald, S. P. Gary, M. F. Thomsen, and H. E. Spence (2009), lon observations from geosynchronous orbit as a proxy for ion cyclotron wave growth during storm times, J. Geophys. Res., 114, A10214, doi:10.1029/2009JA014396.

Chen, L. J., R. M. Thorne, and R. B. Horne (2009), Simulation of EMIC wave excitation in a model magnetosphere including structured highdensity plumes, J. Geophys. Res., 114, A07221, doi:10.1029/2009JA014204.

Cornwall, J. M. (1965), Cyclotron instabilities and electromagnetic emission in the ultra low frequency and very low frequency ranges, J. Geophys. Res., 70(1), 61-69, doi:10.1029/JZ070i001p00061.

Engebretson, M. J., A. Keiling, K. H. Fornacon, C. A. Cattell, J. R. Johnson, J. L. Posch, S. R. Ouick, K. H. Glassmeier, G. K. Parks, and H. Reme (2007), Cluster observations of Pc 1-2 waves and associated ion distributions during the October and November 2003 magnetic storms, Planet. Space Sci., 55(6), 829, doi:10.1016/J.Pss.2006.03.015.

Fraser, B. J., and T. S. Nguyen (2001), Is the plasmapause a preferred source region of electromagnetic ion cyclotron waves in the magnetosphere?, J. Atmos. Sol. Terr. Phys., 63(11), 1225-1247. 
Funsten, H. O., et al. (2013), Helium, Oxygen, Proton, and Electron (HOPE) Mass Spectrometer for the Radiation Belt Storm Probes Mission, Space Sci. Rev., doi:10.1007/s11214-013-9968-7.

Gamayunov, K. V., and G. V. Khazanov (2008), Crucial role of ring current H+ in electromagnetic ion cyclotron wave dispersion relation: Results from global simulations, J. Geophys. Res., 113, A11220, doi:10.1029/2008JA013494.

Gary, S. P., M. B. Moldwin, M. F. Thomsen, D. Winske, and D. J. McComas (1994), Hot proton anisotropies and cool proton temperatures in the outer magnetosphere, J. Geophys. Res., 99(A12), 23,603-23,615, doi:10.1029/94JA02069.

Gurgiolo, C., B. R. Sandel, J. D. Perez, D. G. Mitchell, C. J. Pollock, and B. A. Larsen (2005), Overlap of the plasmasphere and ring current: Relation to subauroral ionospheric heating, J. Geophys. Res., 110, A12217, doi:10.1029/2004JA010986.

Halford, A. J., B. J. Fraser, and S. K. Morley (2010), EMIC wave activity during geomagnetic storm and nonstorm periods: CRRES results, J. Geophys. Res., 115, A12248, doi:10.1029/2010JA015716.

Jordanova, V. K., C. J. Farrugia, R. M. Thorne, G. V. Khazanov, G. D. Reeves, and M. F. Thomsen (2001), Modeling ring current proton precipitation by electromagnetic ion cyclotron waves during the May 14-16, 1997, storm, J. Geophys. Res., 106(A1), 7-22, doi:10.1029/2000JA002008.

Kennel, C. F., and H. E. Petschek (1966), Limit on stably trapped particle fluxes, J. Geophys. Res., 71(1), 1-28, doi:10.1029/JZ071i001p00001. Kessel, R. L, N. J. Fox, and N. Weiss (2013), The Radiation Belt Storm Probes (RBSP) and Space Weather, Space Sci. Rev., doi:10.1007/s11214-012-9953-6,

Khazanov, G. V., K. V. Gamayunov, D. L. Gallagher, and J. U. Kozyra (2007), Reply to comment by R. M. Thorne and R. B. Horne Khazanov et al. [2002] and Khazanov et al. [2006], J. Geophys. Res., 112, A12215, doi:10.1029/2007JA012463.

Kletzing, C. A., et al. (2013), The Electric and Magnetic Field Instrument Suite and Integrated Science (EMFISIS) on RBSP, Space Sci. Rev., 179, 1-4, doi:10.1007/S11214-013-9993-6.

Lin, R.-L., et al. (2014), Testing linear theory of EMIC waves in the inner magnetosphere: Cluster observations, J. Geophys. Res. Space Physics, 119, 1004-1027, doi:10.1002/2013JA019541.

MacDonald, E. A., L. W. Blum, S. P. Gary, M. F. Thomsen, and M. H. Denton (2010), High-speed stream driven inferences of global wave distributions at geosynchronous orbit: Relevance to radiation-belt dynamics, Proc. Roy. Soc. A, 466, 2123, doi:10.1098/rspa.2010.0076.

Mauk, B. H., N. J. Fox, S. G. Kanekal, R. L. Kessel, D. G. Sibeck, and A. Ukhorskiy (2013), Science objectives and rationale for the Radiation Belt Storm Probes Mission, Space Sci. Rev., doi:10.1007/s11214-012-9908-y.

Min, K., J. Lee, K. Keika, and W. Li (2012), Global distribution of EMIC waves derived from THEMIS observations, J. Geophys. Res., 117, A05219, doi:10.1029/2012JA017515.

Pickett, J. S., et al. (2010), Cluster observations of EMIC triggered emissions in association with Pc1 waves near Earth's plasmapause, Geophys. Res. Lett., 37, L09104, doi:10.1029/2010GL042648.

Rauch, J. L., and A. Roux (1982), Ray tracing of ULF waves in a multicomponent magnetospheric plasma: Consequences for the generation mechanism of ion cyclotron waves, J. Geophys. Res., 87(A10), 8191-8198, doi:10.1029/JA087iA10p08191.

Roux, A., S. Perraut, J. L. Rauch, C. Devilledary, G. Kremser, A. Korth, and D. T. Young (1982), Wave-particle interactions near $\Omega \mathrm{He}+$ observed on board GEOS 1 and 2, 2. Generation of ion cyclotron waves and heating of He+ ions, J. Geophys. Res., 87(A10), 8174-8190, doi:10.1029/JA087iA10p08174.

Smith, P. H., and R. A. Hoffman (1974), Direct observations in the dusk hours of the characteristics of the storm time ring current particles during the beginning of magnetic storms, J. Geophys. Res., 79(7), 966-971, doi:10.1029/JA079i007p00966.

Spence, H. E., et al. (2013), Science goals and overview of the Radiation Belt Storm Probes (RBSP) Energetic Particle, Composition, and Thermal Plasma (ECT) Suite on NASA's Van Allen Probes Mission, Space Sci. Rev., doi:10.1007/s11214-013-0007-5.

Thorne, R. M. (2010), Radiation belt dynamics: The importance of wave-particle interactions, Geophys. Res. Lett., 37, L22107, doi:10.1029/ 2010GL044990.

Tsyganenko, N. A., and M. I. Sitnov (2005), Modeling the dynamics of the inner magnetosphere during strong geomagnetic storms, J. Geophys. Res., 110, A03208, doi:10.1029/2004JA010798.

Vallat, C., N. Ganushkina, I. Dandouras, C. P. Escoubet, M. G. G. T. Taylor, H. Laakso, A. Masson, J. A. Sauvaud, H. Reme, and P. Daly (2007), lon multi-nose structures observed by Cluster in the inner magnetosphere, Ann. Geophys., 25, 171-190, doi:10.5194/angeo-25-171-2007.

Young, D. T., S. Perraut, A. Roux, C. Devilledary, R. Gendrin, A. Korth, G. Kremser, and D. Jones (1981), Wave-particle interactions near $\Omega$ He+ observed on GEOS 1 and 2, 1. Propagation of ion cyclotron waves in He+-rich plasma, J. Geophys. Res., 86(A8), 6755-6772, doi:10.1029/JA086iA08p06755.

Zhang, J.-C., L. M. Kistler, C. G. Mouikis, M. W. Dunlop, B. Klecker, and J.-A. Sauvaud (2010), A case study of EMIC-wave associated He+ energization in the outer magnetosphere: Cluster and Double Star 1 observations, J. Geophys. Res., 115, A06212, doi:10.1029/2009JA014784.

Zhang, J. C., L. M. Kistler, C. G. Mouikis, B. Klecker, J. A. Sauvaud, and M. W. Dunlop (2011), A statistical study of EMIC wave-associated He+ energization in the outer magnetosphere: Cluster/CODIF observations, J. Geophys. Res., 116, A11201, doi:10.1029/2011JA016690. 